\title{
Intravascular imaging and novel techniques to disrupt severely calcified lesions
}

\author{
Chinmay Khandkar ${ }^{1,2,3}$, Sanjay Patel ${ }^{2,3,4}$, Keyvan Karimi Galougahi ${ }^{2,3,4}$ \\ ${ }^{1}$ Department of Cardiology, Orange Base Hospital, Orange, Australia \\ ${ }^{2}$ Faculty of Medicine and Health, The University of Sydney, Sydney, New South Wales, Australia, Sydney, Australia \\ ${ }^{3}$ Department of Cardiology Royal Prince Alfred Hospital, Sydney, Australia \\ ${ }^{4}$ Heart Research Institute, Sydney, Australia
}

Adv Interv Cardiol 2021; 17, 4 (66): 337-339

DOI: https://doi.org/10.5114/aic.2021.112523

Advancing age and co-morbid conditions (e.g., diabetes and chronic kidney disease) predispose to significant coronary calcification, which is present in up to one third of patients with coronary artery disease [1]. During percutaneous coronary intervention, these heavily calcified segments can present significant challenges, portending higher risks of both immediate complications (e.g. flow-limiting stent edge dissection) and long-term unfavourable events (e.g. in-stent restenosis (ISR), stent thrombosis, and myocardial infarction) compared with non-calcified lesions. Adjunctive techniques such as rotational atherectomy (RA) and recently intravascular lithotripsy (IVL), used in conjunction with imaging technologies such as intravascular ultrasound (IVUS) and optical computed tomography (OCT), are often necessary in treating heavily calcified lesions.

In this issue of the journal, 3 cases are presented that highlight the complexities of dealing with challenging calcified lesions during percutaneous coronary intervention $(\mathrm{PCl})$. In the first case, RA was used to modify a severely calcified lesion in the right coronary artery (RCA) that was initially resistant to pre-dilation. The ROTABLATOR system (Boston Scientific), which is more than three decades old, utilises rotation of a front-cutting diamond-crusted burr to ablate rigid coronary calcium [2]. Interestingly, the authors in this case identified extensive dissection with OCT imaging when having difficulty with non-compliant (NC) balloon inflations after several runs of a $1.5 \mathrm{~mm}$ burr. As a result, they chose to use a scoring balloon rather than a larger burr to fracture the calcified plaque and allow successful deployment of drug eluting stents. OCT confirmed adequate stent expansion. This case highlights the importance of intracoronary imaging, which provided detailed lesion characterisation for planning of the percutaneous intervention. One important point to highlight is that intracoronary imaging ought to be used upfront when assessing heavily calcified lesions to comprehensively assess the calcium morphology, and thus predict the likelihood of adequate stent expansion, and whether atherectomy devices would be required [1]. This is achieved with OCT by characterising the morphology of calcium deposits as deep, superficial, or nodular. A calcium score is subsequently calculated, which accounts for variables including calcium thickness, angle, and length. A score of $\geq 3$ predicts the need for calcium modification prior to stent deployment, in order to reduce the risk of stent under-expansion. As depicted in Figure 1, this algorithmic approach is our preferred strategy to treat moderate to severely calcified lesions.

In the aforementioned case, the authors avoided using a larger burr with RA due to extensive proximal dissection detected on OCT after atherectomy and NC balloon dilatations. Whilst it is not unreasonable to use alternative modalities such as scoring balloons as in this case, the presence of dissection on OCT is not necessarily an absolute contraindication to further RA. As long as wire position remains in the true lumen, RA can be cautiously applied to safely complete the procedure [3]. Moreover, non-obstructive residual dissections after $\mathrm{PCl}$ detected on OCT only were not associated with major adverse cardiac events or late angiographic/clinical outcomes [4]. It is worth noting that both scoring balloons and NC balloons still pose a risk of dissection when employed to modify calcific plaque [5].

The second case involved the use of IVL under OCT guidance to treat ISR in the setting of incompletely ex-

Corresponding author:

Keyvan Karimi Galougahi MD, PhD, Heart Research Institute, 7 Eliza St, Newtown NSW 2042, Sydney, Australia, phone: +61 282088900 ,

e-mail: Keyvan.karimi@hri.org.au

Received: 29.12.2021, accepted: 29.12.2021. 


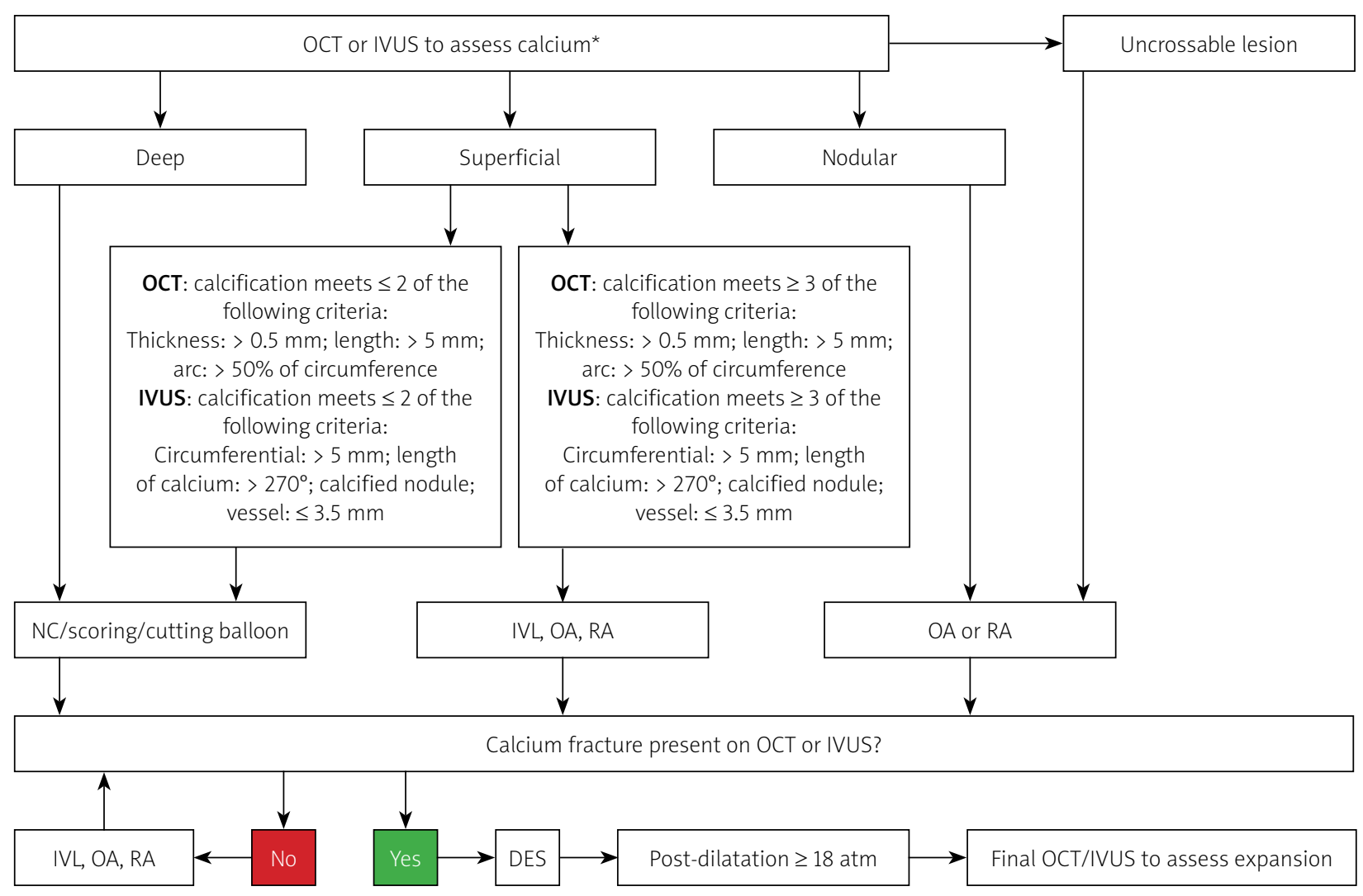

Figure 1. An algorithmic approach for complex calcified lesions. Adapted from Karimi Galougahi et al with permission.

${ }^{*}$ Calcium thickness can only be assessed by optical computed tomography (OCT). IVUS - intravascular ultrasound, NC - non-compliant, IVL - intravascular lithotripsy, OA - orbital atherectomy, RA - rotational atherectomy, DES - drug eluting stent.

panded previous stents at the aorto-ostial segment. Stent under-expansion is a common cause of ISR, implicated in up to $55 \%$ of ISR lesions [1]. IVL delivers sonic pressure waves to fracture calcified plaque, thus increasing lesion compliance and allowing for adequate dilatation of the target lesion [6]. This in turn allows for optimal stent expansion [7]. The authors used IVL to facilitate better expansion, and subsequently used a drug-eluting balloon to deliver an antiproliferative drug. The use of IVL in freshly deployed drug-eluting stents ( $<3$ months from deployment) is not recommended as the shockwaves may damage the stent polymer and lead to incomplete release of the drug [7]. The use of a drug-eluting balloon in this context thus has a plausible rationale. The authors used OCT after the intervention to assess the final result, but again we recommend also using OCT upfront to identify both the extent and aetiology of stent under-expansion and assess for post-IVL fracturing of deep calcium to ensure optimal stent expansion. This step is especially important in this case involving the ostium of the RCA, which is known to be associated with lower procedural success due to marked elastic recoil and neointimal proliferation after balloon expansion [8].

Moreover, the OCT sub-study analysis in Disrupt CAD II [9] suggested that initial assessment with OCT may re- sult in fewer angiographic complications although the differences were not statistically significant. The main advantage of using IVL in the cases of ISR is that it is not affected by the metal scaffolding from the under-expanded stents [7]. Other atherectomy devices such as RA are off-label use and pose the risk of entrapment in the stent struts or vessel perforation. Whilst case series on this relatively novel application of IVL are promising, future trials are required to validate the efficacy and safety of this approach to treating ISR [10]. IVL use in recently implanted (<3 months) under-expanded stents may affect the integrity of the drug polymer coating and increase the risk of ISR due to neointimal proliferation [10]. Whether this risk actually exists and whether the use of drug-eluting balloons can mitigate the risk warrant further studies.

The third case report presents the use of IVL in the setting of recurrent restenosis of an underexpanded stent in a saphenous vein graft (SVG) supplying a second marginal (OM2) artery. The ISR occurred despite multiple balloon angioplasties in previous years with a drug coated balloon. The authors achieved adequate post-dilation with an NC balloon after 80 cycles of shockwaves from IVL. Whilst the use of IVL in this setting remains off-label, it has significant advantages over atherectomy 
techniques such as RA, which poses much higher risks of perforation and distal embolization [7]. The authors highlight that the SVG supplied a relatively large area of myocardium. Given the well-documented increase in risk of no-reflow with $\mathrm{PCl}$ on SVG, one could consider the concurrent use of embolic protection devices to avoid potentially jeopardising the large area of the subtended myocardium in this case [11]. As in the previous cases, we also promote the adjunct use of intracoronary imaging for procedure planning and to confirm optimal stent expansion.

The advent of new percutaneous techniques with high resolution intracoronary imaging has provided the opportunity to approach treatment of heavily calcified lesions known to be high risk of peri-procedural complication. These cases highlight how RA and IVL can be used to good effect in complex cases and provide clinicians with alternative strategies when faced with these challenges.

\section{Conflict of interest}

The authors declare no conflict of interest.

\section{References}

1. Galougahi KK, Shlofmitz E, Jeremias A, et al. Therapeutic approach to calcified coronary lesions: disruptive technologies. Curr Cardiol Rep 2021; 23: 33.

2. Abdel-Wahab M, Richardt G, Büttner HJ, et al. High-speed rotational atherectomy before paclitaxel-eluting stent implantation in complex calcified coronary lesions: the randomized ROTAXUS (Rotational Atherectomy Prior to Taxus Stent Treatment for Complex Native Coronary Artery Disease) trial. JACC Cardiovasc Interv 2013; 6: 10-9.

3. Sharma SK, Tomey MI, Teirstein PS, et al. North American Expert review of rotational atherectomy. Circ Cardiovasc Interv 2019; 12: e007448.

4. Nishida T, Colombo A, Briguori C, et al. Outcome of nonobstructive residual dissections detected by intravascular ultrasound following percutaneous coronary intervention. Am J Cardiol 2002; 89: 1257-62.

5. Tang Z, Bai J, Su SP, et al. Cutting-balloon angioplasty before drug-eluting stent implantation for the treatment of severely calcified coronary lesions. J Geriatr Cardiol 2014; 11: 44-9.

6. Sorini Dini C, Nardi G, Ristalli F, et al. Contemporary approach to heavily calcified coronary lesions. Interv Cardiol 2019; 14: 154 63.

7. Galougahi KK, Patel S, Shlofmitz RA, et al. Calcific plaque modification by acoustic shock waves. Circ Cardiovasc Interv 2021; 14: e009354.

8. Motwani JG, Raymond RE, Franco I, et al. Effectiveness of rotational atherectomy of right coronary artery ostial stenosis. Am J Cardiol 2000; 85: 563-7.

9. Ali ZA, Nef H, Escaned J, et al. Safety and effectiveness of coronary intravascular lithotripsy for treatment of severely calcified coronary stenoses. Circ Cardiovasc Interv 2019; 12: e008434.

10. Yeoh J, Cottens D, Cosgrove C, et al. Management of stent underexpansion using intravascular lithotripsy - defining the utility of a novel device. Catheter Cardiovasc Interv 2021; 97: 22-9.
11. Sturm E, Goldberg D, Goldberg S. Embolic protection devices in saphenous vein graft and native vessel percutaneous intervention: a review. Curr Cardiol Rev 2012; 8: 192-9. 